\title{
INVERSE KINEMATICS AND PID CONTROLLER IMPLEMENTATION OF HEXAPOD ROBOT FOR WALL FOLLOWER NAVIGATION
}

\author{
Riky Tri Yunardi1,*, Arief Muchadin ${ }^{2}$, Kurnia Latifa Priyanti $^{3}$, Deny Arifianto ${ }^{4}$ \\ Departemen Teknik, Fakultas Vokasi, Universitas Airlangga \\ 1,* rikytriyunardi@vokasi.unair.ac.id \\ 2arief.muchadin-2016@vokasi.unair.ac.id \\ ${ }^{3}$ kurnia.latifa.priyanti-2016@vokasi.unair.ac.id \\ 4 denyarifianto23@gmail.com
}

\begin{abstract}
Wall following is one of the methods used in navigating the movement of robot applications. Because the robot moves along the wall, the ultrasonic sensor is used as a barrier detector capable of measuring the distance between the robot and the wall. The six-legged robot is a hexapod robot has six pieces of legs and each leg has three joints that are used to move. The leg movement is based on the inverse kinematics to obtain the angle value of each joint. Nevertheless, a six-legged robot requires stability in order to move smoothly while following the wall. In this work, a robot was developed using a proportional derivative controller to implemented on wall follower navigation. The PID controller is determined using analytic tuning to produce the controller parameters that are used to make the robot move straighten and keep the position against the wall. Overall, the application of inverse kinematics and PID control on the wall following robot navigation can improve the stability of the robot with a set point value of 8-16 cm on the wall length of 1.5 within 92-96 \% of average success rate.
\end{abstract}

Keywords: inverse kinematics, PID controller, hexapod robot, wall follower.

\section{INTRODUCTION}

Mobile robot is a robot designed to move in the area of unstructured and can define their own paths of movement [1]. Based on motion systems, mobile robots are classified into two types are wheeled robot and legged robots. However wheeled robot will have difficulty when used on the uneven ground. While the legged robot can be used more flexibly and able to move in the flat and in the field uneven.

One type of legged robot is the most widely used type of six-legged robot known as a hexapod. Hexapod has many advantages, including flexibility in movement patterns, has better stability when compared to two-legged robots or fourlegged [2]. This current legged robots have been widely used in various applications and research. At the time of the move, one leg swinging upward while the other two legs are still tread on the ground. This is called a static stability, at which the robot load center point is supported by three legs that still tread on the ground [3]. Unlike the two-legged robot, which requires a dynamic balance when moving.

The problem often encountered in the wall follower robot is when the robot runs still often touch or hit the wall and difficult to align the position (parallel) to the wall. As a result, the robot will not be able to run well, reducing the efficiency of time, and can cause damage to the components and mechanics of the robot. Therefore we need a method that the robot can be walked along the wall well is by using wall follower method. Wall follower is one method used for robot navigation [4].

In this study, the wall follower method will be implemented using PD control system. The control system consists of proportional and derivative controller. The elements of the $\mathrm{P}$ controller aims to accelerate the reaction of a system (fast rise time), while the D control aims to reduce overshoot due to the control action P. It will get a system response that has a fast rise time and minimal overshoot [5]. The application of PD algorithm on the wall follower robot will make the robot can navigate more smoothly through the wall [6]. While the PID controller is used on a robot that can move more quickly and stable. This algorithm will be used on a six-legged robot (hexapod) equipped with five pieces of ultrasonic sensors.

\section{WALL FOLLOWER NAVIGATION}

Wall following is one of the methods used to navigate the robot movement based on the wall contours. This method is used by a robot who has the ability to move along the walls or labyrinths to the desired direction by maintaining a constant distance to the wall during walking [7]. Wall following determine the direction of orientation of the robot with the navigation along the wall. One of these advantages is no need for a guide line or a sign as a direction for the robot. This system works by 
adjusting the distance of the wall with the robot to remain constant. If there is a change in distance, the robot will move to then adjust the distance again. Identification to obtain the following robot wall movement is using the left search method and follow the corrugated wall. By providing a set point input the distance between the robot and the wall, proximity sensor mounted on the left side of the robot will measure the distance of the robot as feedback to determine navigation with the desired input. The error value is the difference between the set point value and the measured value used to direct the movement of robot.

\section{MATERIAL AND METHOD}

\section{Design of Six-Legged Robot}

The shape of the foot robot can associate with insect legs [8]. Leg structure consists of three main parts: coxa (hip bone), femur (thigh bone) and tibia (shin bone). At the foot of the robot, the three main parts are connected by three servo motors. Coxa is a part of the hip that functions as a support the mechanical body weight in static and dynamic conditions. The femur is the thigh that serves as a connector between coxa and tibia. Tibia is a shin that connects to the thigh and forming a joint (knee) to serve the robot to walk. The coxa to support forward and backward movement form the leg. The femur supports lifting up and down of a leg. And the tibia support the movements to the right and left of robot. The structure of robot legs shown in the Figure 1.

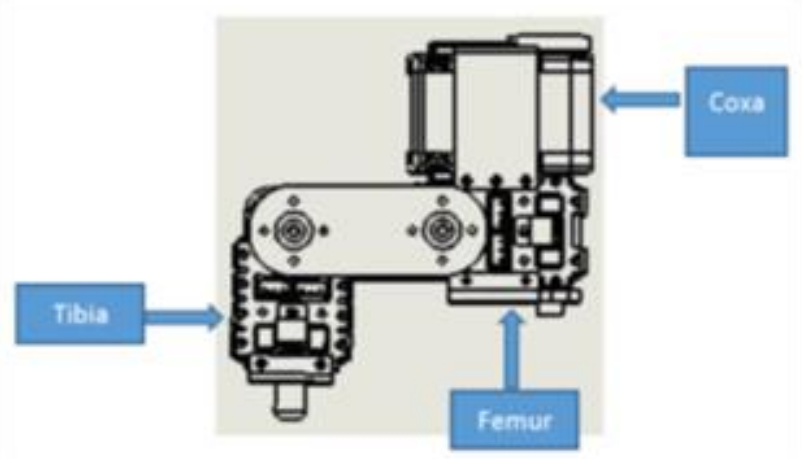

Figure 1. The structure of robot legs

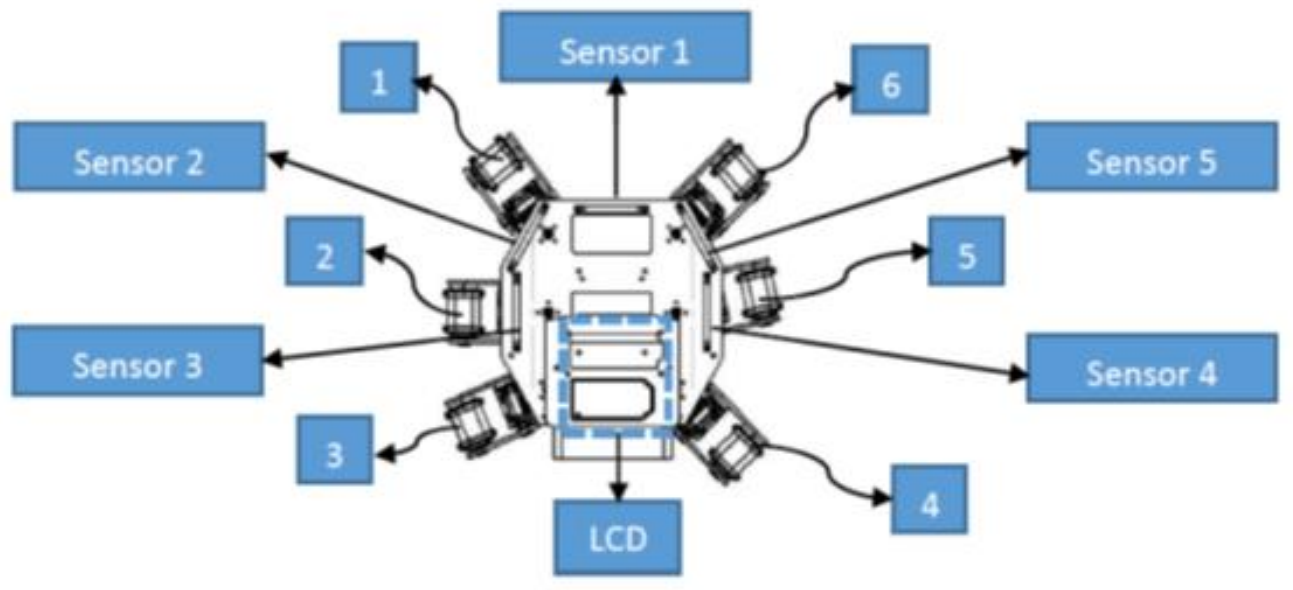

Figure 2. Ultrasonic sensor placement configuration

Ultrasonic sensor placement configuration is shown in Figure 2. The ultrasonic sensor used uses the SRF-04 ultrasonic sensor. The sensor placement design is made up of five sensors, where one sensor is placed in front, two sensors on the left and two sensors on the right side of the robot.

\section{Inverse Kinematics of The Three-Joint Leg}

Inverse kinematic analysis is a method to transform from Cartesian space to joint space. From the kinematic equations, can be obtained relationships between the geometry of the joint space on a leg of the robot coordinate used to determine the desired position [9].
Hexapod leg structure using insect leg structure consists of three joints and three sections of bone, the coxa, femur, and tibia. The three-joint leg structure shown in figure 3 . Inverse kinematics is used to find large joint angles of coxa, femur, and tibia in order to leg the robot can reach the desired end point. The analysis method to obtain the angle value of each joint for coxa $\left(\theta_{1}\right)$, femur $\left(\theta_{2}\right)$ and tibia $\left(\theta_{3}\right)$ angle if the coordinate position $(\mathrm{x}, \mathrm{y}, \mathrm{z})$ must be known.

Based on Figure 3 the end-effector of leg expressed by $P(x, y, z) . P$ is the vector sum consisting of the vector $r_{1}$ (femur) and $r_{2}$ (tibia) is written as follows: 


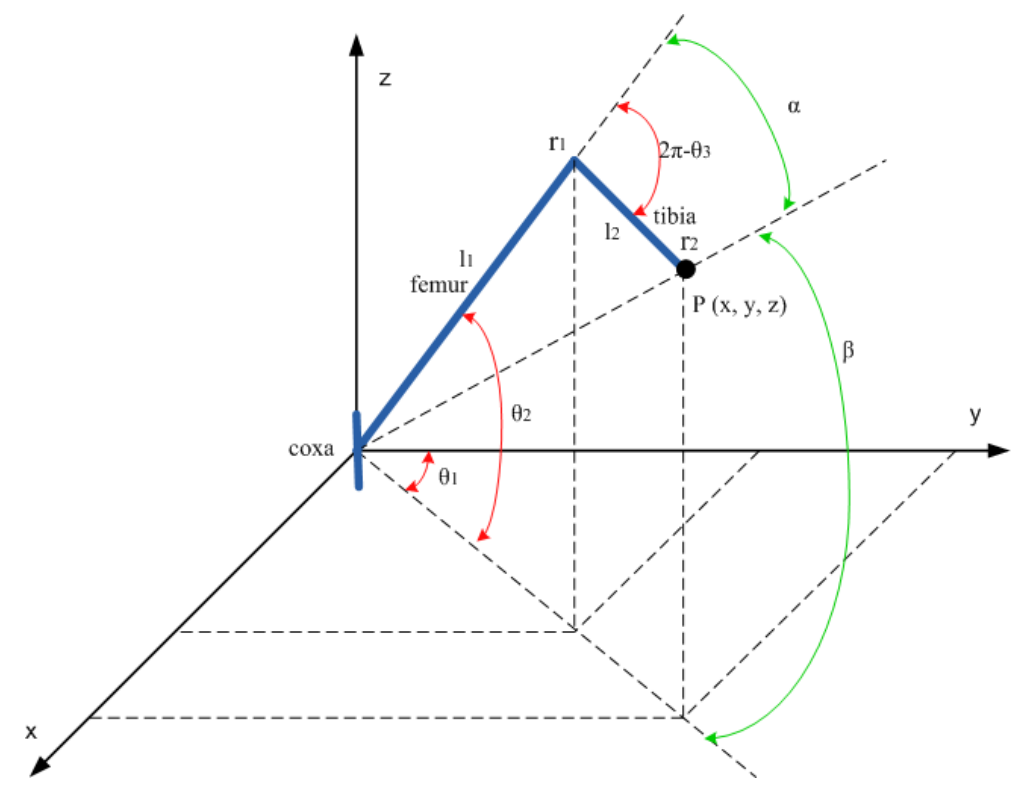

Figure 3. The three-joint leg structure

$r_{1}=\left[l_{1} \sin \theta_{1} \cos \theta_{2}, l_{1} \cos \theta_{1} \cos \theta_{2}, l_{1} \sin \theta_{1}\right]$

$r_{2}=\left[l_{2} \sin \theta_{1} \cos \left(\theta_{2}+\theta_{3}\right), l_{2} \cos \theta_{1} \cos \left(\theta_{2}+\theta_{3}\right)\right.$,

Equation (1) and (2) if it is described in the form of Cartesian coordinates using trigonometry theorems into the equation as follows:

$$
x=l_{1} \sin \theta_{1} \cos \theta_{2}+l_{2} \cos \theta_{1} \cos \theta_{2} \cos \theta_{3}
$$$$
-l_{2} \sin \theta_{1} \sin \theta_{2} \sin \theta_{3}
$$

$y=l_{1} \cos \theta_{1} \cos \theta_{2}+l_{2} \cos \theta_{1} \cos \theta_{2} \cos \theta_{3}$

$$
-l_{2} \cos \theta_{1} \sin \theta_{2} \sin \theta_{3}
$$

$z=l_{1} \sin \theta_{2}+l_{2} \sin \theta_{2} \cos \theta_{3}+l_{2} \sin \theta_{2} \cos \theta_{3}$

In order to get the value of the joint for tibia $\theta_{3}$, where $\sin ^{2} a+\cos ^{2} a=1$, the angle of a servo on tibia can be written using equation (6) and (7).

$\cos \theta_{3}=\frac{x^{2}+y^{2}+z^{2}-l_{1}{ }^{2}-l_{2}{ }^{2}}{2 l_{1} l_{2}}$

$\theta_{3}=\cos ^{-1}\left[\frac{x^{2}+y^{2}+z^{2}-l_{1}{ }^{2}-l_{2}{ }^{2}}{2 l_{1} l_{2}}\right]$
By using trigonometric identity laws, $\tan \theta_{2}=\frac{\tan \alpha+\tan \beta}{1-\tan \alpha \tan \beta}$ where,

$\tan \alpha=\frac{l_{2} \sin \theta_{3}}{l_{2} \cos \theta_{3}+l_{1}}$

$\tan \beta=\frac{z}{\sqrt{x^{2}+y^{2}}}$

The joint for femur $\theta_{2}$ is defined as in equation (10),

$\theta_{2}=\tan ^{-1}\left[\frac{z\left(l_{2} \cos \theta_{3}+l_{1}\right)-\left(\sqrt{x^{2}+y^{2}}\right)\left(l_{2} \sin \theta_{3}\right)}{\left(\sqrt{x^{2}+y^{2}}\right)\left(l_{2} \cos \theta_{3}+l_{1}\right)+z l_{2} \sin \theta_{3}}\right]$

Then on joint for coxa $\theta_{1}$ is derived in equation (11) and equation (12),

$\tan \theta_{1}=\frac{x}{y}$

$\theta_{1}=\tan ^{-1}\left[\frac{x}{y}\right]$ 


\section{PID Control System Design}

PID control is used on the robot to align the position to remain parallel to the wall during the robot move forward. To make the robot move along the wall, the movement will be controlled to move forward, turn left and right. Forward value is a constant value which controls the forward speed moving. Meanwhile, the value of the rotation will be obtained from derivatives proportional control system. This value is used to control the orientation of the robot to make the robot turn left or right. Meanwhile, the value of the rotation will be obtained from the PID control system. This value is used to control the orientation of the robot to make the robot turn left or right. The purpose of detecting this distance is for the robot can walk while maintaining a distance from the wall as far as the set point. PD control system diagram block shown in Fig. 4.

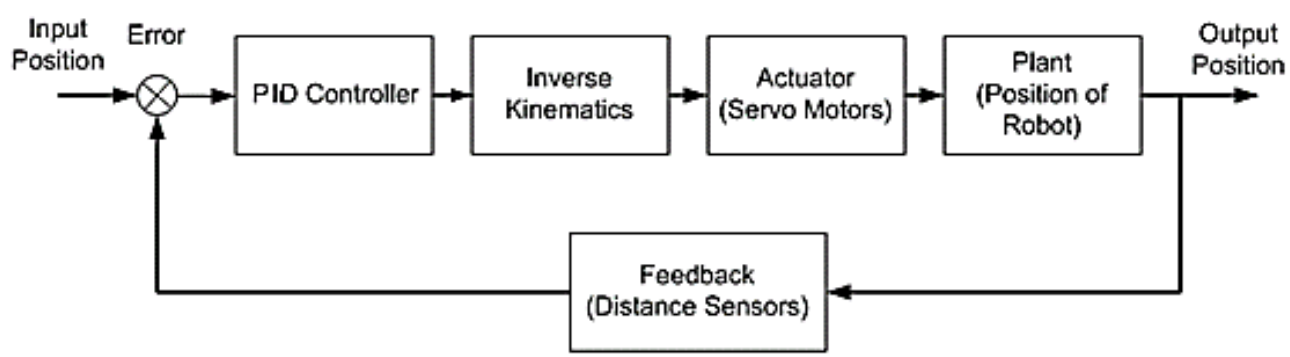

Figure 4. PID control system design

The PID controller is a controller that works in proportion, integral and derivative to the error signal, the relationship between the error signals to the PID controller on the signal can be expressed by equation (13).

PIDvalue $=K_{p} e(t)+K_{i} \int_{0}^{t} e(t) d t+K_{d} \frac{d}{d t} e(t)$

In order to be PID controller discrete form, the PID equation is converted into the form of the transfer function as in equation (14).

$u(k)=K_{p} e(k)+K_{i} T_{s} \sum_{0}^{k} e(k)+K_{d} \frac{1}{T_{s}}\left(e_{k}-e_{k-1}\right)$

Where, $u(k)$ as the output controller, $e(k)$ is an error from the difference between the setpoint with the actual value, $K_{p}$ is proportional constant, $K_{i}$ is integral constant, $K_{d}$ is derivative constant and $T_{S}$ is time sampling.

\section{RESULTS}

\section{The Mechanical Design of Six-Legged Robot}

In this study, the design of a robot created is a six legged robot (hexapod) where at each foot designed with three degrees of freedom (3DOF). DOF is a robotic joints combined with servo motor and built to resemble the legs of insects. The mechanical design of six-legged robot shown in Figure 5.

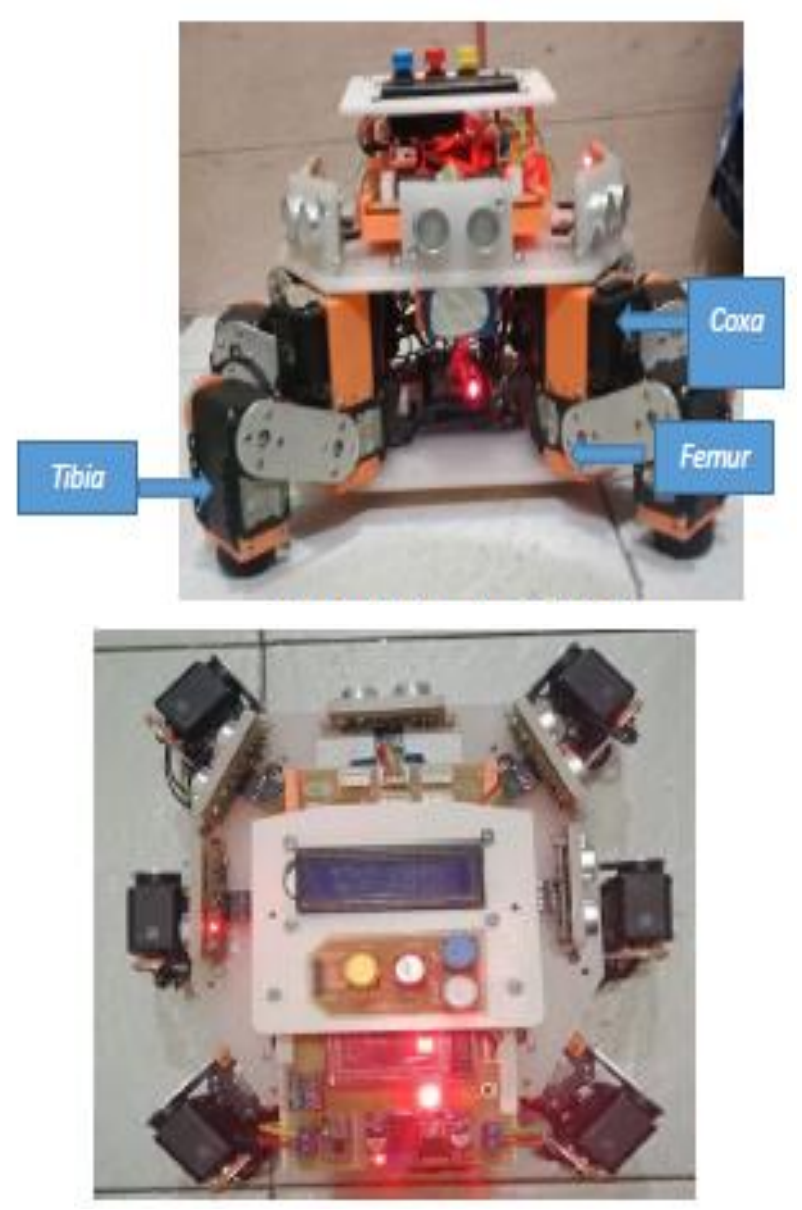

Figure 5 The mechanical design of six-legged robot 


\section{Tuning Parameter PID}

Controller parameter tuning is a method to obtain the controller parameter corresponding to the desired response. Determining the value of PID parameters were obtained by trial and error. This is done many times in order to get the desired response and provide value of the $K_{p}, K_{i}$ and,$K_{d}$ in control of PID. Based on the results obtained by tuning the value of $K_{p}=25, K_{i}=0.5$ and $K_{d}=1$ by means of test control parameters that possess the most excellent system response.

\section{Testing the Robot Motion}

Forward movement on the robot, there are two stages. The first stage, leg 2, leg 4 and leg 6 moves forward while legs 1, leg 3 and leg 5 remain stationary in the initial position. The second stage of leg 1, leg 3 and leg 5 to moves forward and the leg 2, leg 4, leg 6 remain in the last position. In forward movement, coxa moves toward the front of the servo position on the femur, in order that part of the tibia does not touch the surface of the floor. After the coxa position is appropriate, the femur descends and makes the tibia touch the floor. Further, when the condition of coxa back into the starting position, the femur part will hold the tibia in order to land that makes the robot will move forward. Illustration of forward movement on the robot shown in Figure 6.
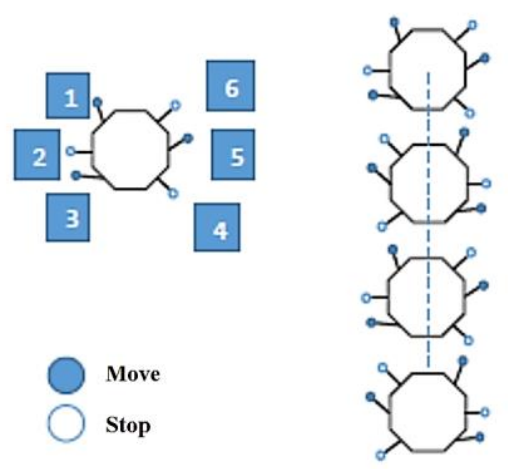

Figure 6. Illustration of forward movement on the robot
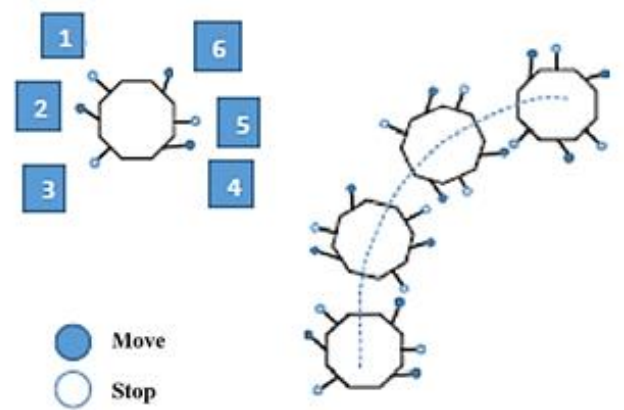

Figure 7. Illustration of robot turn right
In Figure 7 shown for illustration of robot turn right, the robot moves with the position of the left coxa change position by 45 degrees and coxa right by 10 degrees.

For a left turn, the servo left has a larger rotational movement and servo right has a small rotational movement of the position. From testing the motion to the right, the robot makes a right turn to the direction toward 90 degrees. The position of the right leg section coxa of the robot moves by 45 degrees and left at 10 degrees. On the femur and tibia same as the process moves forward motion, where to turn right, at the servo of coxa, has a bigger role to make the robot position change. Illustration of robot turn left shown in Figure 8 .
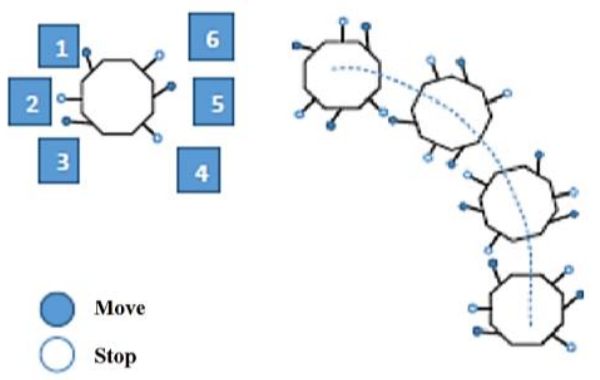

Figure 8. Illustration of robot turn left

\section{Testing Robot Followed Wall Left Side}

In Figure 9 shows the trajectory resulting from motion robot to variable distances. Robots were instructed to follow the left wall. At a distance of $8 \mathrm{~cm}$ from the wall, the resulting unstable trajectories because at the time during robot walking, the robot moves to the right before stabilizing. At a distance of $10 \mathrm{~cm}$ from the wall, the robot runs very unstable and causes the motion is not smooth. At a distance of $12 \mathrm{~cm}, 14 \mathrm{~cm}$ and $16 \mathrm{~cm}$ robot turn right then walk stably along the wall. This can be because of the distances that are too far away from the wall surface and the error value produced is also too large.

\section{CONCLUSION}

The inverse kinematics to obtain the angle value of each joint. The PID controller used to make the robot move straighten and keep the position against the wall. Based on test results that look by applying inverse kinematics and PID control, the robot successfully improves the stability of the robot with a set point value of $8-16 \mathrm{~cm}$ on the wall length of 1.5 within $92-96 \%$ of the average success rate. The condition of walls and obstacles in the path greatly affects the performance of sensors that affect the movement of the robot is generated. Testing and testing is still to be done, especially in determining its tuning PID controller, necessitating the robot can navigate without involving or hit the wall. 


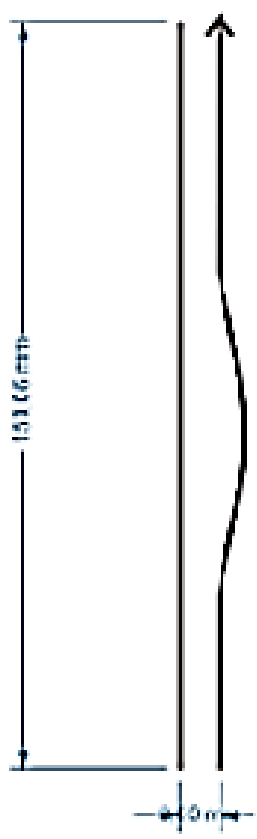

(a) $S \mathrm{~cm}$

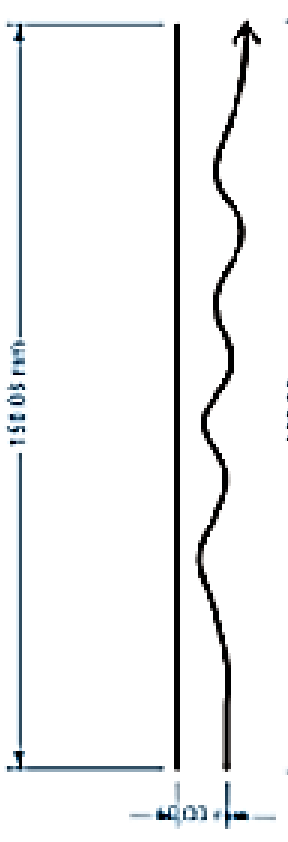

(b) $10 \mathrm{~cm}$

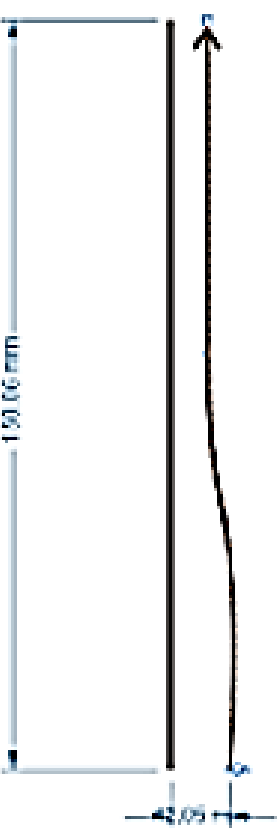

(c) $12 \mathrm{~cm}$

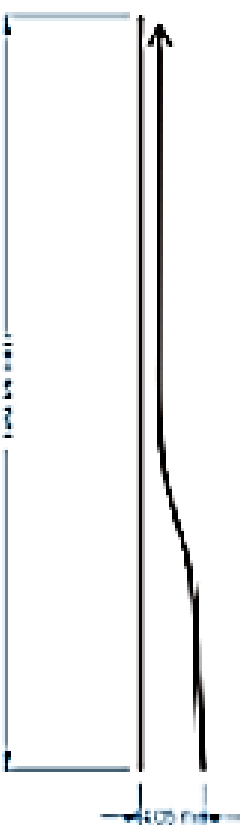

(d) $14 \mathrm{~cm}$

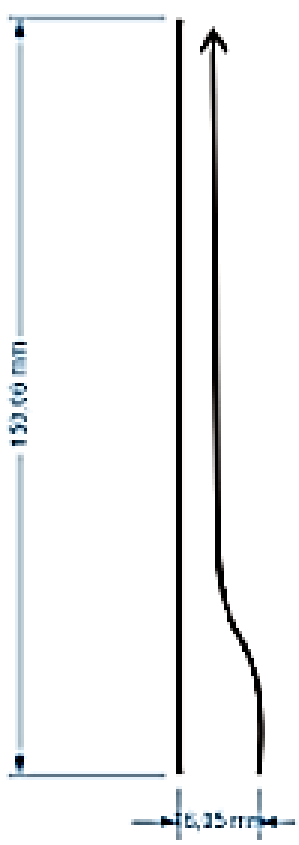

(e) $16 \mathrm{~cm}$

Figure 9. The trajectory resulting from motion robot

\section{REFERENCES}

[1] Silver, David, J. Andrew Bagnell, and Anthony Stentz. "Learning from demonstration for autonomous navigation in complex unstructured terrain." The International Journal of Robotics Research 29, no. 12 (2010): 1565-1592.

[2] Deng, Hua, Guiyang Xin, Guoliang Zhong, and Michael Mistry. "Object carrying of hexapod robots with integrated mechanism of leg and arm." Robotics and Computer-Integrated Manufacturing 54 (2018): 145-155.

[3] Erden, Mustafa Suphi, and Kemal Leblebicioğlu. "Free gait generation with reinforcement learning for a sixlegged robot." Robotics and Autonomous Systems 56, no. 3 (2008): 199-212.

[4] Hanafi, Dirman, Yousef Moh Abueejela, and Mohamad Fauzi Zakaria. "Wall follower autonomous robot development applying fuzzy incremental controller." Journal of Intelligent Control and Automation (ICA) 4 (1) (2013): 18-25.

[5] Wang, Jie-Sheng, Yong Zhang, and Wei Wang. "Optimal design of PI/PD controller for non-minimum phase system." Transactions of the Institute of Measurement and Control 28, no. 1 (2006): 27-35.

[6] Carelli, Ricardo, and Eduardo Oliveira Freire. "Corridor navigation and wall-following stable control for sonarbased mobile robots." Robotics and Autonomous Systems 45, no. 3-4 (2003): 235-247.
[7] Poncela, Alberto, Cristina Urdiales, Eduardo J. Pérez, and Francisco Sandoval. "A new efficiency-weighted strategy for continuous human/robot cooperation in navigation." IEEE Transactions on Systems, Man, and Cybernetics-Part A: Systems and Humans 39, no. 3 (2009): 486-500.

[8] Weidemann, H-J., Friedrich Pfeiffer, and Jürgen Eltze. "The six-legged TUM walking robot." In Proceedings of IEEE/RSJ International Conference on Intelligent Robots and Systems (IROS'94), vol. 2, pp. 1026-1033. IEEE, 1994. 